\title{
Thickness measurement of circular metallic film using single-frequency eddy current sensor
}

\author{
Mingyang $\mathrm{Lu}^{\mathrm{a}, 1,{ }^{*} \text {, Xiaobai Meng }}{ }^{\mathrm{b}, 1}$, Ruochen Huang ${ }^{\mathrm{a},{ }^{*}, \text { Liming Chen }}{ }^{\mathrm{a}}$, Anthony Peytona ${ }^{\mathrm{a}}$, Wuliang Yin $^{\mathrm{a}, *}$ \\ ${ }^{a}$ School of Electrical and Electronic Engineering, University of Manchester, Oxford Road, Manchester, M13 9PL, UK \\ ${ }^{\mathrm{b}}$ Faculty of Art, Science and Technology, University of Northampton, Northampton, NN1 5PH, UK \\ ${ }^{1} \mathrm{M}$. Lu and X. Meng contributed equally and share the first authorship. \\ *Corresponding author: Mingyang.lu@ manchester.ac.uk; ruocheng.huang@manchester.ac.uk; wuliang.yin@manchester.ac.uk;
}

\begin{abstract}
In many advanced industrial applications, the thickness is a critical index, especially for metallic coatings. However, the variance of lift-off spacing between sensors and test pieces affects the measured voltage or impedance, which leads to unreliable results from the sensor. Massive research works have been proposed to address the lift-off issue, but few of them applies to the thickness measurement of planar metallic films with finitesize circular (disk) geometry. Previously, a peak-frequency feature from the swept-frequency inductance was used to compensate the measurement error caused by lift-offs, which was based on the slow-changing rate of impedance phase term in the Dodd-Deeds formulas. However, the phase of measured impedance is nearly invariant merely on a limited range of sample thicknesses and working frequencies. Besides, the frequency sweeping is timeconsuming, where a recalibration is needed for different sensor setups applied to the online real-time measurement. In this paper, a single-frequency algorithm has been proposed, which is embedded in the measurement instrument for the online real-time retrieval of thickness. Owing to the single-frequency measurement strategy, the proposed method does not need to recalibrate for different sensor setups. The thickness retrieval is based on a triple-coil sensor (with one transmitter and two receivers). The thickness of metallic disk foils is retrieved from the measured electrical resistance of two transmitter-receiver sensing pairs. Experiments on materials of different electrical conductivities (from direct current), thicknesses and planar sizes (radii) have been carried out to verify the proposed method. The error for the thickness retrieval of conductive disk foils is controlled within $5 \%$ for liftoffs up to $5 \mathrm{~mm}$.
\end{abstract}

Index Terms - Eddy current testing, thickness measurement, finite-size, lift-off effect, non-destructive testing.

\section{Introduction}

Electromagnetic (EM) eddy current testing (ECT) [1-7] is one of promising technologies in the non-destructive testing, which applies to the evaluation of product quality (e.g., inspecting the crack fatigue, measuring the inhomogeneity or monitoring the variance of the property) [8-14]. The thickness measurement system has the potential application of inspecting the structural integrity and surface notches of metals, particularly for conductive coatings. Both the multi-frequency eddy current sensors, swept-frequency eddy current sensors, and pulsed eddy current (PEC) sensors have been used for the thickness measurement of metallic plates or foils (e.g., coatings or claddings) [15-24]. However, it is found that the variance of sample-sensor lift-offs significantly affects the measured voltage or impedance of sensors, which influences the accuracy on thickness retrieval.

Many techniques have been used for eliminating the lift-off effect when using both the multi-frequency eddy current sensors, swept-frequency eddy current sensors, and pulsed eddy current (PEC) sensors, including custom embedded algorithms, new sensor designs, and novel time-domain and frequency-domain features. Tai et al. have introduced a custom-built instrument for determining the thickness and electrical conductivity of coatings using PEC sensors [21]. Wang et al. have proposed a novel feature from the slope of the lift-off curve for reducing the lift-off error on the thickness measurement of metallic films [22]. Pinotti et al. proposed a lock-in method to extract the phase signature and linked it to the electrical conductivity and thickness of conductive coatings [23]. Mandache et al. have found a lift-off point of intersection (LOI) feature in the transient and harmonic eddy currents [24]. Fan et al. have used the LOI feature for reducing the lift-off effect on the thickness measurement when using the PEC method [25]. Angani et al. have used the LOI feature for the detection of corrosion and thickness measurement of stainless-steel plates [26]. These methods are efficient on the thickness measurement but cannot apply to the thickness measurement of finite-size circular plates.

In our previous research works, various techniques have been proposed for reducing the lift-off effect, including the peak frequency feature in swept-frequency inductance spectra, and novel sensor designs for the single-frequency testing [27-32]. The peak frequency feature is based on the slow-changing rate of impedance phase term in the Dodd-Deeds model. However, the changing rate of impedance phase term is neglectable merely on a limited range of sample thicknesses. 
A single-frequency algorithm is proposed in this paper, which is used to reduce the lift-off effect on retrieving the thickness of metallic films with finite-size circular geometry. The algorithm is based on the modified DoddDeeds model [30, 34]. Instead of integrating from zero (for the infinite plate) [33], for the case of finite-size disk sample, the algorithm of mutual impedance is revised by integrating from a constant value in the Dodd-Deeds model. By combining the single-frequency impedance measured from two transmitter-receiver sensing pairs, the thickness of conductive disk foils is retrieved and less affected by the lift-off of sensors. Experiments on finite disk plates with different electrical conductivities, actual thicknesses and radii have been carried out for the validation of the proposed algorithm.

\section{Methodology - algorithm for the thickness measurement of no-ferrous metal plate with finite-size circular geometry}

Both the lift-off of sensors and properties of samples (including the thickness, magnetic permeability, electrical conductivity) affect the detected signal (voltage, mutual impedance, or inductance) of the electromagnetic eddy current sensor. However, the lift-off (or lift-off variance) of sensors is an unknown parameter in most of occasions and applications. Besides, the sensor response is very sensitive to its lift-off to the sample. Thus, in Fig. 1, for the thickness measurement of a non-ferrous circular metal plate, an air coil sensor with two sensing pairs has been designed to address the issue caused by sensor lift-offs.

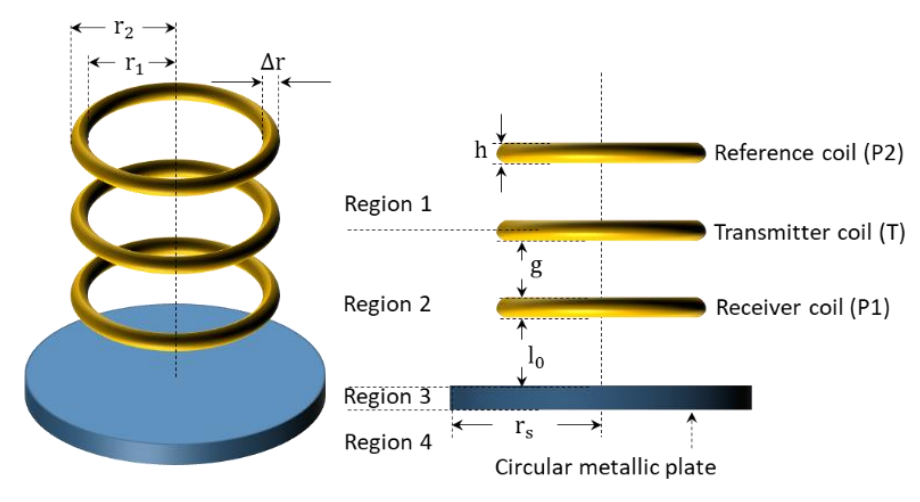

Fig. 1 Three coils co-axially above a finite-size circular plate

Dodd-Deeds analytical model has been proposed over a few decades but is still the most dominant method for the problem of a coil above an infinite plate [33].

From our previous research works, the modified Dodd-Deeds model has been proposed for the problem of circular coils above a finite-size circular disk plate [30, 34]. By integrating the previous derived magnetic vector potential in region 1 and 2, the impedance change (subtract of the impedance for the presence and absence of the specimen) tested by two sensing pairs can be derived as,

$$
\begin{aligned}
& \Delta \mathrm{Z}_{1}=\mathrm{j} \omega \overline{\mathrm{r} K} \int_{\alpha_{\mathrm{t}}}^{\infty} \frac{\mathrm{P}(\alpha)}{\alpha^{6}} \mathrm{~A}_{1}(\alpha) \phi(\alpha) \mathrm{d} \alpha \\
& \Delta \mathrm{Z}_{2}=\mathrm{j} \omega \overline{\mathrm{r} K} \int_{\alpha_{\mathrm{t}}}^{\infty} \frac{\mathrm{P}(\alpha)}{\alpha^{6}} \mathrm{~A}_{2}(\alpha) \phi(\alpha) \mathrm{d} \alpha
\end{aligned}
$$

$\omega$ is the working angular frequency. $\mathrm{K}$ is a coil factor and defined in (3). $\alpha$ is related to the wavenumber of incident transverse electric (TE) planar electromagnetic wave [33-43]. $\mathrm{P}(\alpha)$ is the integration of Bessel terms defined in (10). $A_{1}(\alpha)$ and $A_{2}(\alpha)$ are the coil-dependent term defined in (8) and (9). $\phi(\alpha)$ is a complex term determined by the test piece (i.e. circular metallic plate), and defined in (6).

$$
\mathrm{K}=\frac{\pi \mathrm{N}^{2} \mu_{0}}{\mathrm{~h}^{2} \Delta \mathrm{r}^{2}}
$$

$\mathrm{N}$ and $\mathrm{h}$ are the identical turns and height of the transmitter coil (T), receiver coil (P1) and reference coil (P2). $\mu_{0}$ is the vacuum permeability. $\overline{\mathrm{r}}$ and $\Delta \mathrm{r}$ are the mean radii and difference between the outer radius $\mathrm{r}_{2}$ and inner radius $r_{1}$ of three coils, which is defined in (4).

$$
\overline{\mathrm{r}}=\frac{\mathrm{r}_{1}+\mathrm{r}_{2}}{2}, \Delta \mathrm{r}=\frac{\mathrm{r}_{2}-\mathrm{r}_{1}}{2}
$$


$\alpha_{t}$ is the lower limit of the integration for the finite-size circular film, which is related to the radius of disk (circular) plate $r_{s} \cdot\left(\alpha_{t}\right.$ is zero for an infinite plate)

$$
\begin{gathered}
\alpha_{\mathrm{t}}=\frac{3.518}{\mathrm{r}_{\mathrm{s}}} \\
\phi(\alpha)=\frac{\left(\alpha_{1}+\mu_{1} \alpha\right)\left(\alpha_{1}-\mu_{1} \alpha\right)-\left(\alpha_{1}+\mu_{1} \alpha\right)\left(\alpha_{1}-\mu_{1} \alpha\right) \mathrm{e}^{2 \alpha_{1} \mathrm{c}}}{-\left(\alpha_{1}-\mu_{1} \alpha\right)\left(\alpha_{1}-\mu_{1} \alpha\right)+\left(\alpha_{1}+\mu_{1} \alpha\right)\left(\alpha_{1}+\mu_{1} \alpha\right) \mathrm{e}^{2 \alpha_{1} \mathrm{c}}}
\end{gathered}
$$

$\mu_{1}$ and $c$ are the relative permeability and thickness of the tested metallic plate.

$$
\alpha_{1}=\sqrt{\alpha^{2}+j \omega \sigma \mu_{1} \mu_{0}}
$$

$j$ is the imaginary unit. $\sigma$ is the electrical conductivity of the tested piece.

$$
\begin{gathered}
A_{1}(\alpha)=e^{-\alpha\left(2 l_{0}+g+h\right)}\left(e^{-\alpha h}-1\right)^{2} \\
A_{2}(\alpha)=e^{-\alpha\left(2 l_{0}+3 g+3 h\right)}\left(e^{-\alpha h}-1\right)^{2}
\end{gathered}
$$

$\mathrm{g}$ is the gap between coils (T, P1, and P2). $\mathrm{l}_{0}$ is the lift-off between sensor and sample.

$$
P(\alpha)=\int_{\alpha \mathrm{r}_{1}}^{\alpha \mathrm{r}_{2}} \tau \mathrm{J}_{1}(\tau) \mathrm{d} \tau
$$

In (10), $\mathrm{J}_{1}$ is the first-order Bessel function of the first kind. $\tau$ is the integration variable with lower and upper limits of $\alpha r_{1}$ and $\alpha r_{2}$ respectively.

The phase term $\phi(\alpha)$ can be split into its real part $\operatorname{Re}(\phi(\alpha))$ and imaginary part $\operatorname{Im}(\phi(\alpha))$. Therefore, substitute $A_{1}$ in (1) with (8),

$$
\begin{gathered}
\operatorname{Re}\left(\Delta \mathrm{Z}_{1}\right)=-\omega \overline{\mathrm{r}} \mathrm{K} \int_{\alpha_{\mathrm{t}}}^{\infty} \frac{\mathrm{P}(\alpha)}{\alpha^{6}} \mathrm{e}^{-\alpha\left(2 \mathrm{l}_{0}+\mathrm{g}+\mathrm{h}\right)}\left(\mathrm{e}^{-\alpha \mathrm{h}}-1\right)^{2} \operatorname{Im}(\phi(\alpha)) \mathrm{d} \alpha \\
\operatorname{Im}\left(\Delta \mathrm{Z}_{1}\right)=\omega \overline{\mathrm{r}} \mathrm{K} \int_{\alpha_{\mathrm{t}}}^{\infty} \frac{\mathrm{P}(\alpha)}{\alpha^{6}} \mathrm{e}^{-\alpha\left(2 \mathrm{I}_{0}+\mathrm{g}+\mathrm{h}\right)}\left(\mathrm{e}^{-\alpha \mathrm{h}}-1\right)^{2} \operatorname{Re}(\phi(\alpha)) \mathrm{d} \alpha
\end{gathered}
$$

Similarly, substitute $A_{2}$ in (2) with (9),

$$
\begin{gathered}
\operatorname{Re}\left(\Delta \mathrm{Z}_{2}\right)=-\omega \overline{\mathrm{r}} \mathrm{K} \int_{\alpha_{\mathrm{t}}}^{\infty} \frac{\mathrm{P}(\alpha)}{\alpha^{6}} \mathrm{e}^{-\alpha\left(2 \mathrm{I}_{0}+3 \mathrm{~g}+3 \mathrm{~h}\right)}\left(\mathrm{e}^{-\alpha \mathrm{h}}-1\right)^{2} \operatorname{Im}(\phi(\alpha)) \mathrm{d} \alpha \\
\operatorname{Im}\left(\Delta \mathrm{Z}_{2}\right)=\omega \overline{\mathrm{r} K} \int_{\alpha_{\mathrm{t}}}^{\infty} \frac{\mathrm{P}(\alpha)}{\alpha^{6}} \mathrm{e}^{-\alpha\left(2 \mathrm{I}_{0}+3 \mathrm{~g}+3 \mathrm{~h}\right)}\left(\mathrm{e}^{-\alpha \mathrm{h}}-1\right)^{2} \operatorname{Re}(\phi(\alpha)) \mathrm{d} \alpha
\end{gathered}
$$

The phase term $\phi(\alpha)$ was directly taken out of the integration from the previous research works [10]. However, both the real and imaginary part of the phase term $\phi(\alpha)$ is found to be significant especially under low frequencies. Therefore, it is worth to investigate a new general feature of the phase term $\phi(\alpha)$ for a wide range of frequencies.

For the non-ferrous plate, as shown in Fig. 2, the ratio between the imaginary part of the phase term $\operatorname{Im}(\phi(\alpha))$ and real part of the phase term multiplied by $\alpha-\alpha \operatorname{Re}(\phi(\alpha))$ is found to be a constant for a single working frequency.

$$
\frac{\operatorname{Im}(\phi(\alpha))}{\alpha \operatorname{Re}(\phi(\alpha))}=\mathrm{Y}(\omega)
$$




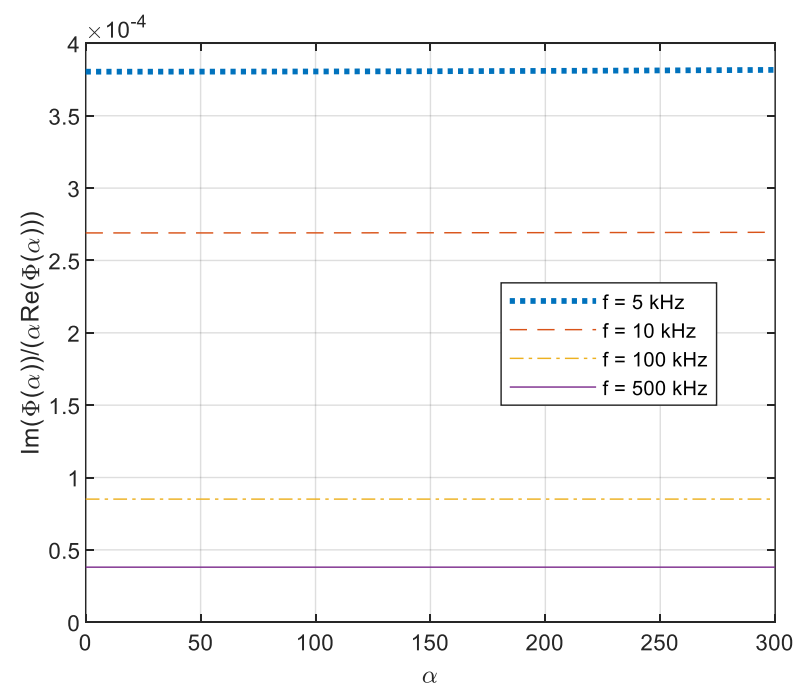

Fig. $2 \frac{\operatorname{Im}(\phi(\alpha))}{\alpha \operatorname{Re}(\phi(\alpha))}$ versus $\alpha$ under different working frequencies

The frequency-dependent constant Y can be calculated by finding its limit at $\alpha=0$.

$$
\mathrm{Y}=\lim _{\alpha \rightarrow 0} \frac{\operatorname{Im}(\phi(\alpha))}{\alpha \operatorname{Re}(\phi(\alpha))}
$$

Equation (16) can be solved using Wolfram, as shown in equation (17).

$$
Y=\frac{\sqrt{2}\left(1+\mathrm{e}^{2 \mathrm{c} \sqrt{2 \omega \sigma \mu_{0}}}\right)}{\sqrt{\omega \sigma \mu_{0}}\left(\mathrm{e}^{2 \mathrm{c} \sqrt{2 \omega \sigma \mu_{0}}}-2 \mathrm{e}^{\mathrm{c} \sqrt{2 \omega \sigma \mu_{0}}} \cos \left(\mathrm{c} \sqrt{2 \omega \sigma \mu_{0}}\right)+1\right)}
$$

Since term $c \sqrt{2 \omega \sigma \mu_{0}}$ is not significant for the thin film, equation (17) can be simplified using the Padé approximation.

$$
\mathrm{Y}=\frac{1}{\sqrt{2 \omega \sigma \mu_{0}}}\left(1+\frac{1}{\mathrm{c}^{2} \omega \sigma \mu_{0}}\right)
$$

As shown in Fig. 3, the real part of the phase term $\phi(\alpha)$ is found to be varying slowly with $\alpha$. In (19), an exponential function with its power controlled by a small factor $G$ is used to approximate $\operatorname{Re}(\phi(\alpha))$. The factor $\mathrm{G}$ is determined by the working frequency $\omega$, electrical conductivity $\sigma$, and sample thickness $\mathrm{c}$.

$$
\operatorname{Re}(\phi(\alpha))=-\mathrm{e}^{-2 \alpha \mathrm{G}}
$$

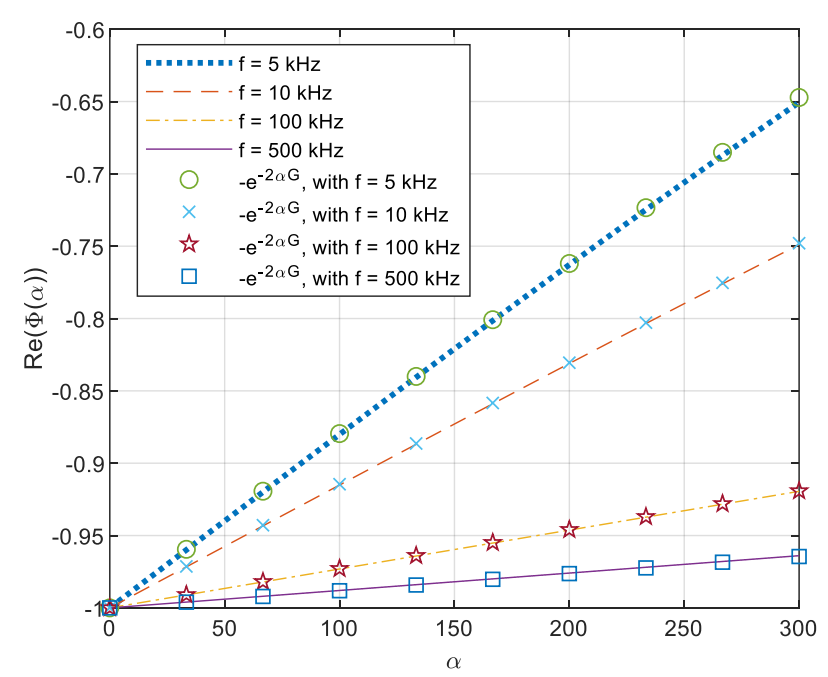

Fig. 3 Approximation of $\operatorname{Re}(\phi(\alpha))$ using $-\mathrm{e}^{-2 \alpha G}$ under different working frequencies 
Assign $\operatorname{Re}\left(\Delta \mathrm{Z}_{1}\right)$ and $\operatorname{Re}\left(\Delta \mathrm{Z}_{2}\right)$ to be $\mathrm{R}_{1}$ and $\mathrm{R}_{2}$. Substitute (15) and (19) into (11)-(14), the electrical resistance change (real parts) and imaginary parts of the impedance change from T-P1 and T-P2 sensing pairs are,

$$
\begin{gathered}
\mathrm{R}_{1}=\omega \mathrm{Yr} \mathrm{K} \int_{\alpha_{\mathrm{t}}}^{\infty} \frac{\mathrm{P}(\alpha)}{\alpha^{5}} \mathrm{e}^{-\alpha\left(2 \mathrm{I}_{0}+\mathrm{g}+\mathrm{h}\right)}\left(\mathrm{e}^{-\alpha \mathrm{h}}-1\right)^{2} \mathrm{e}^{-2 \alpha \mathrm{G}} \mathrm{d} \alpha \\
\operatorname{Im}\left(\Delta \mathrm{Z}_{1}\right)=-\omega \overline{\mathrm{r} K} \int_{\alpha_{\mathrm{t}}}^{\infty} \frac{\mathrm{P}(\alpha)}{\alpha^{6}} \mathrm{e}^{-\alpha\left(2 \mathrm{I}_{0}+\mathrm{g}+\mathrm{h}\right)}\left(\mathrm{e}^{-\alpha \mathrm{h}}-1\right)^{2} \mathrm{e}^{-2 \alpha \mathrm{G}} \mathrm{d} \alpha \\
\mathrm{R}_{2}=\omega \mathrm{Y} \overline{\mathrm{r} K} \int_{\alpha_{\mathrm{t}}}^{\infty} \frac{\mathrm{P}(\alpha)}{\alpha^{5}} \mathrm{e}^{-\alpha\left(2 \mathrm{I}_{0}+3 \mathrm{~g}+3 \mathrm{~h}\right)}\left(\mathrm{e}^{-\alpha \mathrm{h}}-1\right)^{2} \mathrm{e}^{-2 \alpha \mathrm{G}} \\
\operatorname{Im}\left(\Delta \mathrm{Z}_{2}\right)=-\omega \overline{\mathrm{r} K} \int_{\alpha_{\mathrm{t}}}^{\infty} \frac{\mathrm{P}(\alpha)}{\alpha^{6}} \mathrm{e}^{-\alpha\left(2 \mathrm{I}_{0}+3 \mathrm{~g}+3 \mathrm{~h}\right)}\left(\mathrm{e}^{-\alpha \mathrm{h}}-1\right)^{2} \mathrm{e}^{-2 \alpha \mathrm{G}} \mathrm{d} \alpha
\end{gathered}
$$

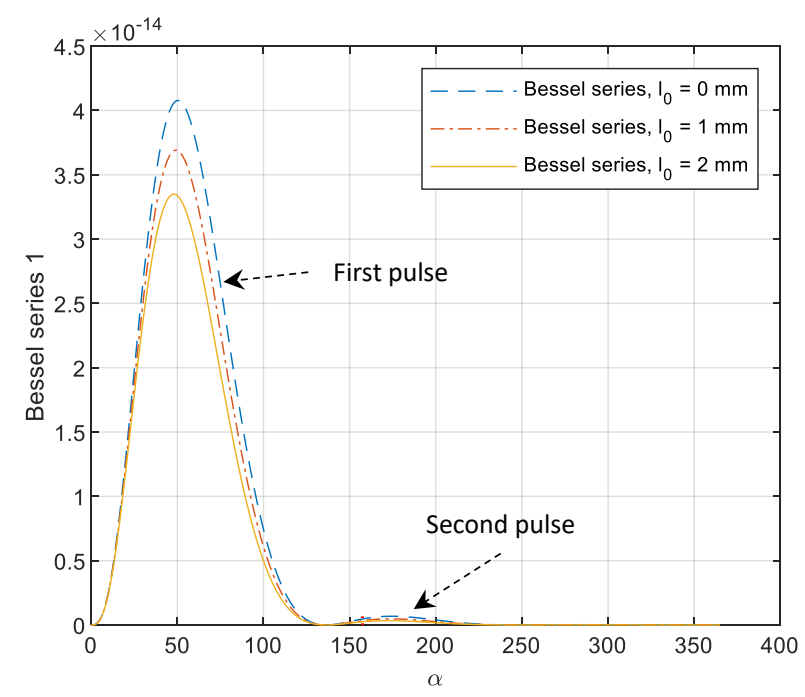

(a)

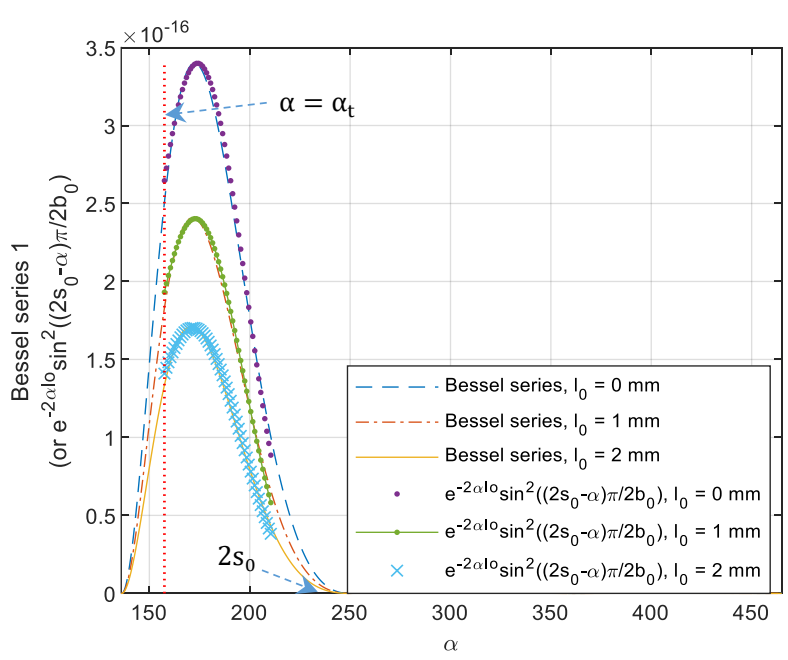

(b)

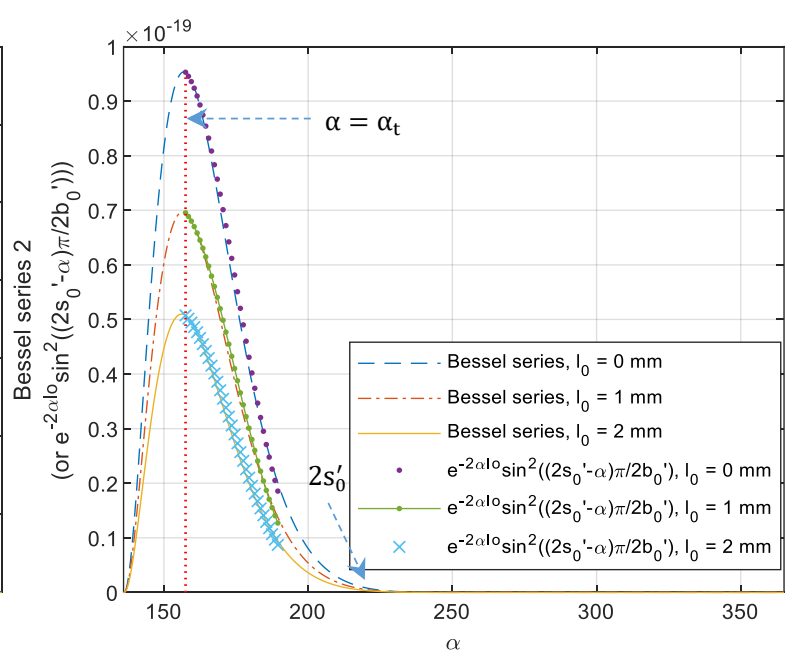

(c)

Fig. 4 (a) The first pulse and second pulse of Bessel series for T-P1 sensing pair (b) Approximation of second-pulse Bessel series for T-P1 sensing pair (c) Approximation of second-pulse Bessel series for T-P2 sensing pair

As Fig. 4 (b) and (c) depict, the Bessel series in (20) and (22) can be estimated as squared sinusoidal functions, as shown in followings, 


$$
\begin{aligned}
\mathrm{R}_{1} & =\omega \mathrm{Y} \overline{\mathrm{r} K} \int_{\alpha_{\mathrm{t}}}^{\infty} \frac{\mathrm{P}(\alpha)}{\alpha^{5}} \mathrm{e}^{-\alpha\left(2 \mathrm{l}_{0}+\mathrm{g}+\mathrm{h}\right)}\left(\mathrm{e}^{-\alpha \mathrm{h}}-1\right)^{2} \mathrm{e}^{-2 \alpha \mathrm{G}} \\
& =\omega \mathrm{Y} \overline{\mathrm{r} K M} \mathrm{M}_{1} \int_{\alpha_{\mathrm{t}}}^{2 \mathrm{~s}_{0}} \mathrm{e}^{-2 \alpha\left(\mathrm{l}_{0}+\mathrm{G}\right)} \sin ^{2}\left(\frac{\left(2 \mathrm{~s}_{0}-\alpha\right) \pi}{2 \mathrm{~b}_{0}}\right) \mathrm{d} \alpha \\
\mathrm{R}_{2} & =\omega \mathrm{Y} \overline{\mathrm{r} K} \int_{\alpha_{\mathrm{t}}}^{\infty} \frac{\mathrm{P}(\alpha)}{\alpha^{5}} \mathrm{e}^{-\alpha\left(2 \mathrm{l}_{0}+3 \mathrm{~g}+3 \mathrm{~h}\right)}\left(\mathrm{e}^{-\alpha \mathrm{h}}-1\right)^{2} \mathrm{e}^{-2 \alpha \mathrm{G}} \\
& =\omega \mathrm{Y} \overline{\mathrm{r} K M} \mathrm{~K}_{1} \int_{\alpha_{\mathrm{t}}}^{2 \mathrm{~s}_{0}^{\prime}} \mathrm{e}^{-2 \alpha\left(\mathrm{l}_{0}+\mathrm{G}\right)} \sin ^{2}\left(\frac{\left(2 \mathrm{~s}_{0}^{\prime}-\alpha\right) \pi}{2 \mathrm{~b}_{0}^{\prime}}\right) \mathrm{d} \alpha
\end{aligned}
$$

In (24) and (25), both $\mathrm{s}_{0}, \mathrm{~b}_{0}, \mathrm{~s}_{0}^{\prime}$, and $\mathrm{b}_{0}^{\prime}$ are used to approximate the Bessel terms in (20) and (22), which are determined by the sensor geometry. The variables $\mathrm{s}_{0}, \mathrm{~b}_{0}, \mathrm{~s}_{0}^{\prime}$, and $\mathrm{b}_{0}^{\prime}$ are similar to the spatial frequency proposed in previous research works $[10,29]$. The spatial frequency in previous work is used to approximate the first pulse of the Bessel series. However, since $\alpha_{t}$ is located on the second pulsed of Bessel series (for a finite-size circular plate), more spatial frequency parameters (variables $s_{0}, b_{0}, s_{0}^{\prime}$, and $b_{0}^{\prime}$ ) are needed for approximating the peak position and bandwidth of the second pulse of Bessel series. As can be seen from Fig. 4 (b) and (c), $s_{0}$ and $s_{0}^{\prime}$ control the upper limit of the integration. Besides, $b_{0}$ and $b_{0}^{\prime}$ define the peak value points of the sinusoidal functions. $\mathrm{M}_{1}$ and $\mathrm{M}_{2}$ are the normalization factor between the Bessel and sinusoidal functions for both T-P1 and T-P2 sensing pairs, which are defined as followings,

$$
\begin{aligned}
& M_{1}=\frac{P\left(2 s_{0}-b_{0}\right)}{\left(2 s_{0}-b_{0}\right)^{5}} e^{-\left(2 s_{0}-b_{0}\right)(g+h)}\left(e^{-\left(2 s_{0}-b_{0}\right) h}-1\right)^{2} \\
& M_{2}=\frac{P\left(2 s_{0}^{\prime}-b_{0}^{\prime}\right)}{\left(2 s_{0}^{\prime}-b_{0}^{\prime}\right)^{5}} e^{-3\left(2 s_{0}^{\prime}-b_{0}^{\prime}\right)(g+h)}\left(e^{-\left(2 s_{0}^{\prime}-b_{0}^{\prime}\right) h}-1\right)^{2}
\end{aligned}
$$

After the integration, equation (24) becomes,

$$
\begin{gathered}
\mathrm{R}_{1}=\frac{\omega \mathrm{Y}_{\bar{r} K M_{1}}}{4\left(\mathrm{l}_{0}+\mathrm{G}\right)\left(4 \mathrm{~b}_{0}^{2}\left(\mathrm{l}_{0}+\mathrm{G}\right)^{2}+\pi^{2}\right)}\left(\mathrm { e } ^ { - 2 \alpha _ { \mathrm { t } } ( \mathrm { l } _ { 0 } + \mathrm { G } ) } \left(4 \mathrm{~b}_{0}^{2}\left(\mathrm{l}_{0}+\mathrm{G}\right)^{2}\right.\right. \\
\left.\left.\left(1-\cos \left(\frac{\left(2 \mathrm{~s}_{0}-\alpha_{\mathrm{t}}\right) \pi}{\mathrm{b}_{0}}\right)\right)+2 \pi \mathrm{b}_{0}\left(\mathrm{l}_{0}+\mathrm{G}\right) \sin \left(\frac{\left(2 \mathrm{~s}_{0}-\alpha_{\mathrm{t}}\right) \pi}{\mathrm{b}_{0}}\right)+\pi^{2}\right)-\pi^{2} \mathrm{e}^{-4 \mathrm{~s}_{0}\left(\mathrm{l}_{0}+\mathrm{G}\right)}\right)
\end{gathered}
$$

Since $b_{0}\left(l_{0}+G\right) \ll \pi,(28)$ becomes,

$$
\mathrm{R}_{1}=\omega \mathrm{Y} \overline{\mathrm{r}} \mathrm{KM}_{1} \frac{\mathrm{e}^{-2 \alpha_{\mathrm{t}}\left(\mathrm{l}_{0}+\mathrm{G}\right)}-\mathrm{e}^{-4 \mathrm{~s}_{0}\left(\mathrm{l}_{0}+\mathrm{G}\right)}}{4\left(\mathrm{l}_{0}+\mathrm{G}\right)}
$$

According to the Padé approximation, $\mathrm{e}^{-2 \alpha_{\mathrm{t}}\left(\mathrm{l}_{0}+\mathrm{G}\right)}$ and $\mathrm{e}^{-4\left(\alpha_{0}+\mathrm{b}_{0}\right)\left(\mathrm{l}_{0}+\mathrm{G}\right)}$ in (29) can be approximate as,

$$
\begin{gathered}
\mathrm{e}^{-2 \alpha_{\mathrm{t}}\left(\mathrm{l}_{0}+\mathrm{G}\right)}=-\frac{\alpha_{\mathrm{t}}\left(\mathrm{l}_{0}+\mathrm{G}\right)-1}{\alpha_{\mathrm{t}}\left(\mathrm{l}_{0}+G\right)+1} \\
\mathrm{e}^{-4\left(\alpha_{0}+\mathrm{b}_{0}\right)\left(\mathrm{l}_{0}+\mathrm{G}\right)}=-\frac{2 \mathrm{~s}_{0}\left(\mathrm{l}_{0}+\mathrm{G}\right)-1}{2 \mathrm{~s}_{0}\left(\mathrm{l}_{0}+G\right)+1}
\end{gathered}
$$

Substitute (30) and (31) into (29), $\mathrm{R}_{1}$ becomes,

$$
\mathrm{R}_{1}=\omega \mathrm{YrKM}_{1} \frac{\left(2 \mathrm{~s}_{0}-\alpha_{\mathrm{t}}\right)}{2\left(\alpha_{\mathrm{t}}\left(\mathrm{l}_{0}+\mathrm{G}\right)+1\right)\left(2 \mathrm{~s}_{0}\left(\mathrm{l}_{0}+\mathrm{G}\right)+1\right)}
$$

Similarly, the resistance from T-P2 sensing pair is

$$
\mathrm{R}_{2}=\omega \mathrm{Y} \overline{\mathrm{r}} \mathrm{KM}_{2} \frac{\left(2 \mathrm{~s}_{0}^{\prime}-\alpha_{\mathrm{t}}\right)}{2\left(\alpha_{\mathrm{t}}\left(\mathrm{l}_{0}+\mathrm{G}\right)+1\right)\left(2 \mathrm{~s}_{0}^{\prime}\left(\mathrm{l}_{0}+\mathrm{G}\right)+1\right)}
$$

Combine (32) with (33), the proportional factor $Y$ is,

$$
Y=\frac{R_{1} R_{2}\left(M_{2} R_{1}-M_{1} R_{2}\right)\left(2 s_{0}^{2}\left(2 s_{0}^{\prime}-\alpha_{t}\right)+2 s_{0}^{\prime 2}\left(\alpha_{t}-2 s_{0}\right)+\alpha_{t}^{2}\left(s_{0}-s_{0}^{\prime}\right)\right)}{\omega \bar{r} K\left(R_{1} s_{0} M_{2}\left(2 s_{0}^{\prime}-\alpha_{t}\right)-R_{2} s_{0}^{\prime} M_{1}\left(2 s_{0}-\alpha_{t}\right)\right)^{2}}
$$


Combine (34) with (18), the thickness of the circular film can be reconstructed as,

$$
c=\frac{1}{\sqrt{\omega \sigma \mu_{0} \sqrt{2 \omega \sigma \mu_{0}} Y-1}}
$$

\section{Experimental verification}

As shown in Fig. 5, experiments have been conducted on the mutual impedance measurement of an air-core sensor co-axially deployed (with lift-offs from $1 \mathrm{~mm}$ to $5 \mathrm{~mm}$ ) above circular aluminium and copper foils. As listed in Table 1, the sensor has three coils with identical parameters, with one transmitter coil, one receiver coil, and one reference coil spirally wound and equally separated on the same cylindrical tube (plastic).

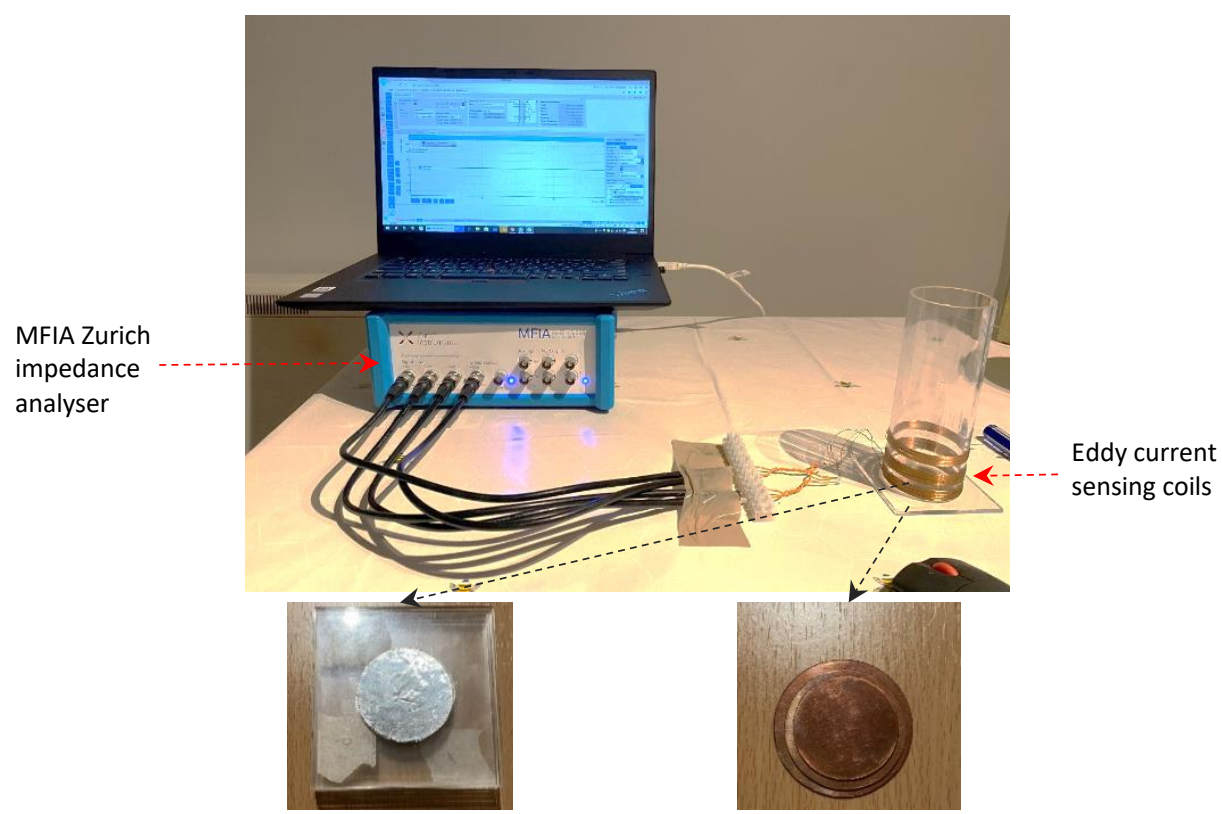

Fig. 5 Experimental configuration - eddy current coils are co-axially placed above aluminium and copper disk laminates with different liftoffs; Impedance analyser is connected to the sensing coil to measure the mutual impedance under different working frequencies of alternating currents flowing in coils.

Table 1 Sensor Parameters

\begin{tabular}{cc}
\hline & T, P1, P2 coils \\
\hline Inner radius $(\mathrm{mm})$ & 28 \\
Outer radius $(\mathrm{mm})$ & 28.25 \\
Turns & 20 \\
Coils gap $(\mathrm{mm})$ & 3.00 \\
Coils height $(\mathrm{mm})$ & 5.00 \\
Lift-offs $(\mathrm{mm})$ & $1.00: 1.00: 5.00$ \\
\hline \hline
\end{tabular}

Table 2 Geometry and property of the metallic films

\begin{tabular}{ccc}
\hline \hline & Aluminium & Copper \\
\hline Radius (mm) & 20.0 & $15.0,17.5,20.0$ \\
Actual thickness & $22,44,66 \mu \mathrm{m}$ & $0.5 \mathrm{~mm}$ \\
$\begin{array}{c}\text { Electrical conductivity } \\
(\mathrm{MS} / \mathrm{m})\end{array}$ & 35 & 57 \\
\hline \hline
\end{tabular}

As listed in Table 2, the aluminium films have an identical radius of $20 \mathrm{~mm}$, but different actual thicknesses. While the copper films have different radii but an identical thickness of $0.5 \mathrm{~mm}$. The electrical conductivity of both samples is measured using the 4-terminal sensing method. Both samples are selected for testing the accuracy of the proposed method on different parameters (radius, thickness, and electrical conductivity). 
In the measurement, three coils of sensor were connected to the impedance analyser. To test the performance of proposed method under different single frequencies, the measurement was tested under a reasonable range of working frequencies from $1 \mathrm{kHz}$ to $500 \mathrm{kHz}$ (to get rid of the noise signal and resonance distortion under lower and higher frequencies).

\section{Results and discussions}

\section{A. Measurement of resistance}

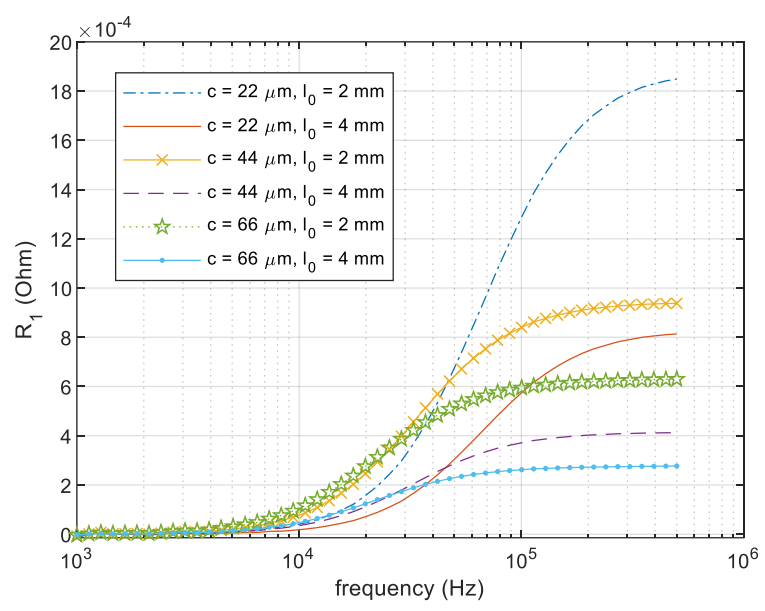

(a)

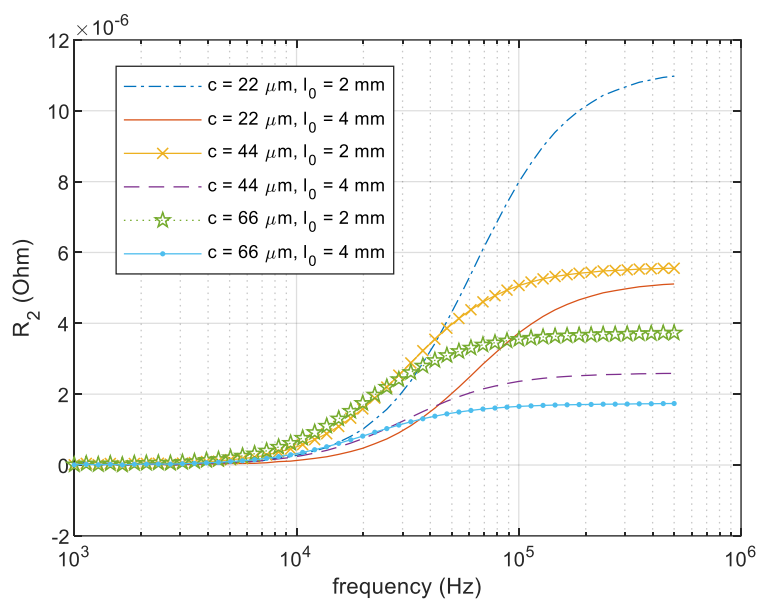

(b)

Fig. 6 Measurement of electrical resistance for the aluminium film of 22, 44, and $66 \mu \mathrm{m}$ under a sensor lift-off of 2 and 4 mm (a) T-P1 sensing pair (b) T-P2 sensing pair

Fig. 6 and Fig. 7 illustrate the measured electrical resistance under different working frequencies and sensor lift-offs $(2 \mathrm{~mm}$ and $4 \mathrm{~mm}$ ). For both aluminium films with different actual thicknesses and copper films with different radii, the measured resistance is shown to be increasing with working frequencies. The measured resistance for a sensor above a metallic sample is related to the skin depth of the eddy current flowing in the sample. Under the high frequencies, the eddy current is restrained underneath the surface of the sample, which is named as the eddy current skin effect or the eddy current diffusion effect. Moreover, due to the weakened interaction between the sensor and sample, the increase in sensor lift-off leads to reduced resistance. Consequently, the measured resistance of T-P2 sensing pair is lower than that of T-P1 sensing pair. As Fig. 6 depicts, under high working frequencies, the resistance of thin aluminium films is larger than that of thicker films. This is because the eddy current flowing in the thin film is more restrained under its lower surface. However, further increased frequency will result in overlap for the curves (over $15 \mathrm{MHz}$ for skin depth of less than $22 \mu \mathrm{m}$, not shown in Fig. 6) of different thicknesses due to the eddy current skin effect. In Fig. 7, the measured resistance for the copper film of a larger radius is larger than that of a smaller radius due to more volume of resistant eddy current or fortified coupling effect between the sensor and sample.

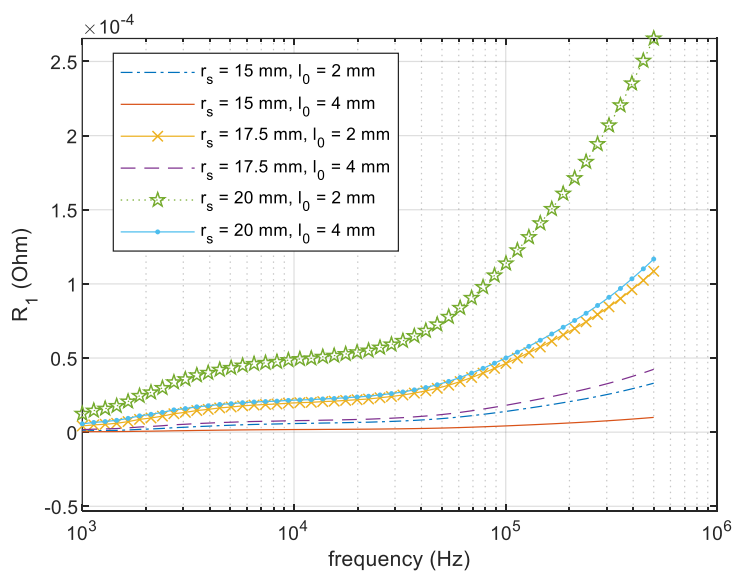

(a)

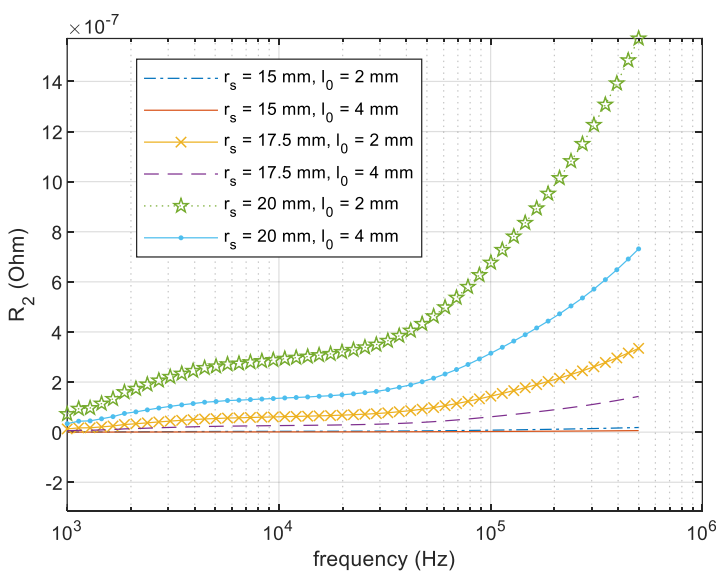

(b)

Fig. 7. Measurement of electrical resistance for the copper film with radii of $15 \mathrm{~mm}, 17.5 \mathrm{~mm}$ and 20 mm under a sensor lift-off of 2 and 4 $\mathrm{mm}$ (a) T-P1 sensing pair (b) T-P2 sensing pair 


\section{B. Reconstructed thickness for different working frequencies}

The thickness of both the aluminium film (with different actual thickness) and copper film (with different radii) can be reconstructed by inputting the measured electrical resistance into (34) and (35). As shown in Fig. 8, for both materials, the reconstructed thickness was found to be reduced with increased single operation frequency. It can be observed that the optimal single operation frequency (using the designed sensor) for both materials is around $16 \mathrm{kHz}$ (since the variables $\mathrm{s}_{0}, \mathrm{~b}_{0}, \mathrm{~s}_{0}^{\prime}$, and $\mathrm{b}_{0}^{\prime}$ in 24,25 , and 34 are fitted at this frequency). The distorted thickness in Fig. 8 for frequencies apart from the optimal value $(16 \mathrm{kHz})$ is mainly due to the discrepancy of coil windings between the experimental and analytical setups. In the analytical setup, the coil winding is modelled as seamless turns of coils with cubic cross-section; while in the experiment, the coils are wound with wires of circular cross-section. Besides, the variation of frequency could result in different off-set impedance due to the fringe effect of current flowing in turns of coil. Thus, for an alternative single frequency (small enough to ensure that the skin depth is larger than the actual thickness), the variables $s_{0}, b_{0}, s_{0}^{\prime}$, and $b_{0}^{\prime}$ in 24,25 , and 34 need to be refitted. In addition, the effect of the sensor lift-off (a small lift-off of less than $5 \mathrm{~mm}$ ) is significantly reduced for the derived thickness (both aluminium with different thicknesses and copper films with different radii), with the curve of $5 \mathrm{~mm}$ sensor lift-off slightly larger than that of $1 \mathrm{~mm}$ sensor lift-off. Moreover, the reconstructed thickness for the copper film is approaching zero (distorted) under high frequencies, which is due to the reduced skin depth (lower than the actual thickness $-0.5 \mathrm{~mm}$ when the frequency is larger than $18 \mathrm{kHz}$ ). Besides, in Fig. 8 (b), it can be seen that the reconstructed thickness is almost immune to the different radii.

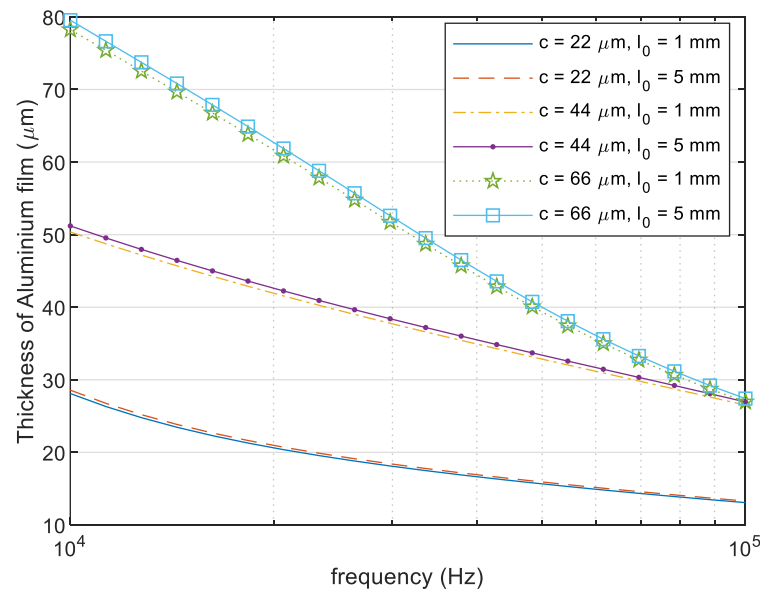

(a)

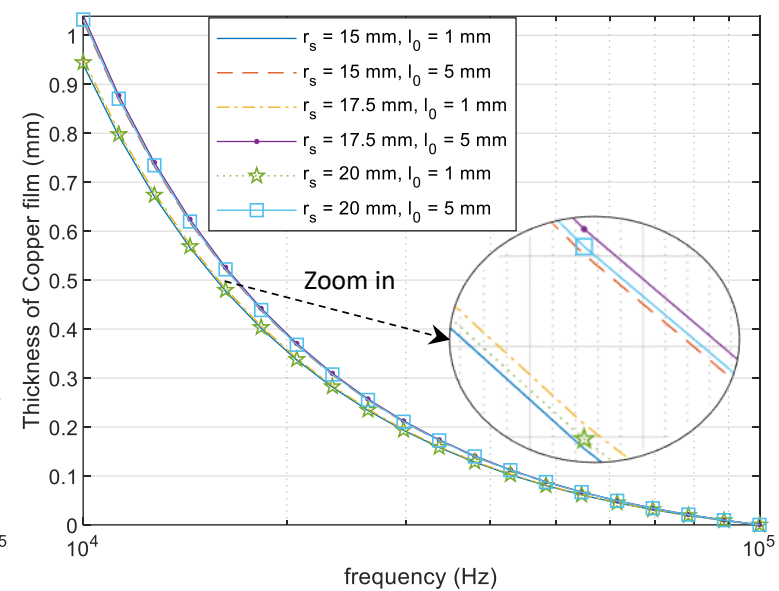

(b)

Fig. 8 Reconstructed thickness under different operating single-frequencies with sensor lift-off of 1 and $5 \mathrm{~mm}$ (a) aluminium film with a thickness of 22, 44, and $66 \mu \mathrm{m}$ (b) copper film with radii of 15, 17.5, and $20 \mathrm{~mm}$

C. Wobble effect between sample and coil
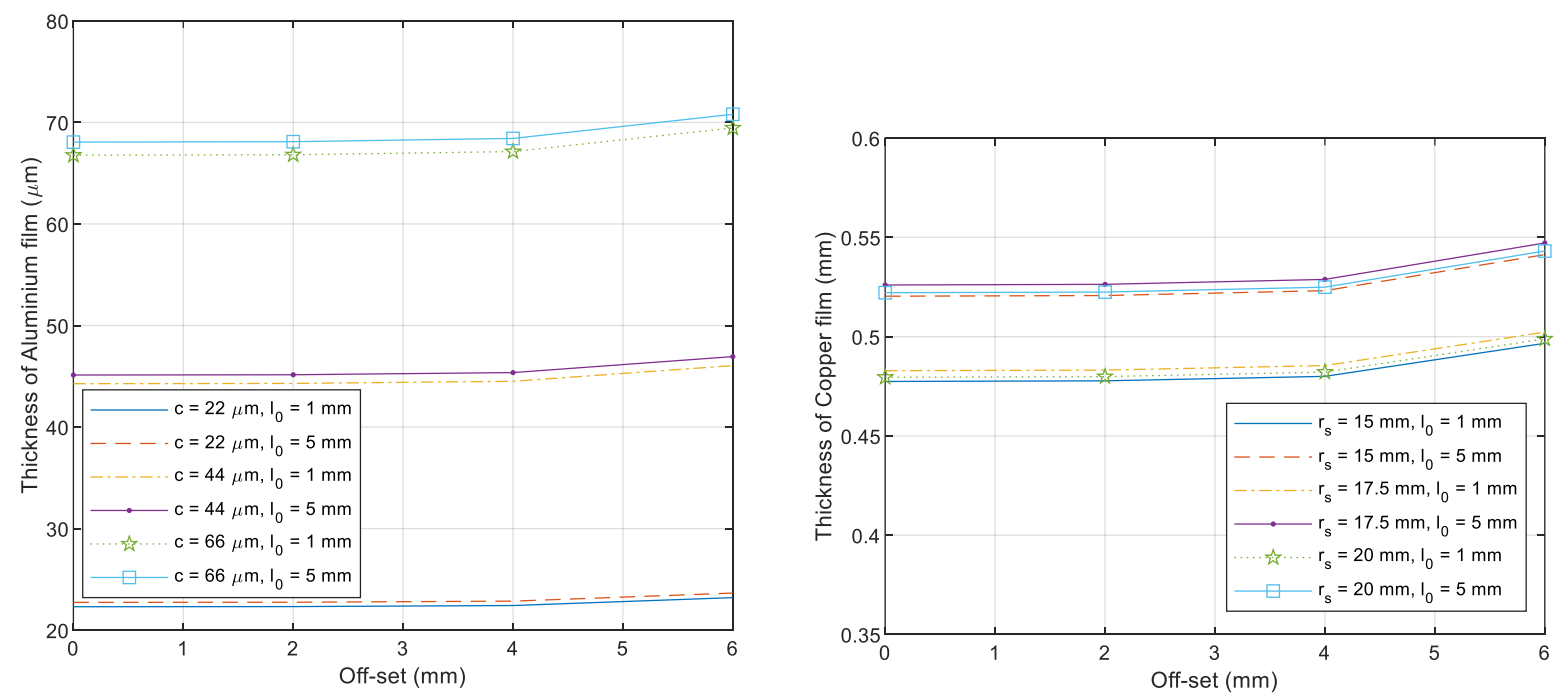

Fig. 9 Reconstructed thickness under different offsets between the axis of sample and coils with sensor lift-off of 1 and 5 mm (a) aluminium film with a thickness of 22, 44, and $66 \mu \mathrm{m}$ (b) copper film with radii of $15,17.5$, and $20 \mathrm{~mm}$ 
Fig. 9 shows the retrieved thickness for different offsets between the axis of sample and coils. It can be observed that a wobble between the sample and coils results in an increased retrieved thickness, which is due to the reduced electrical resistance measured from coils. For a maximum offset of $6 \mathrm{~mm}$, the error of retrieved thickness for both materials are controlled within $10 \%$. Further offsets between the axis of sample and coils will lead to a significantly increased error of thickness retrieval.

\section{Reconstructed thickness for different sensor lift-offs}

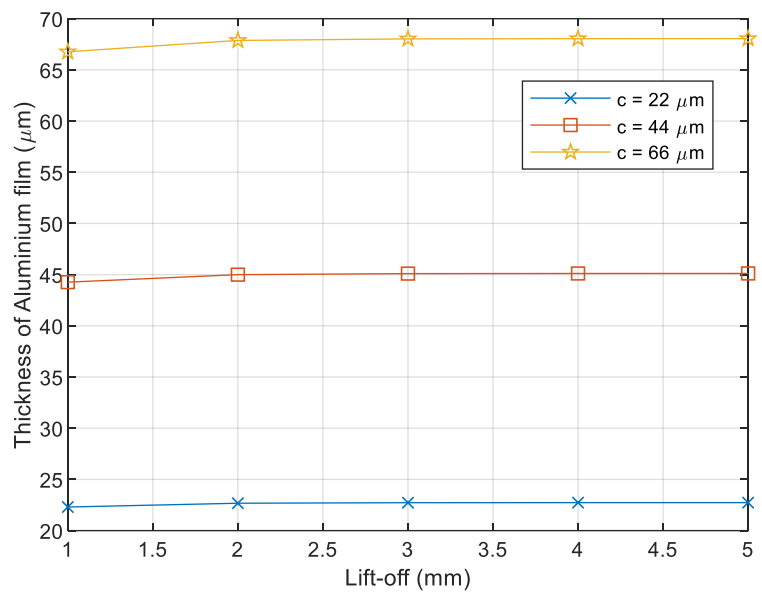

(a)

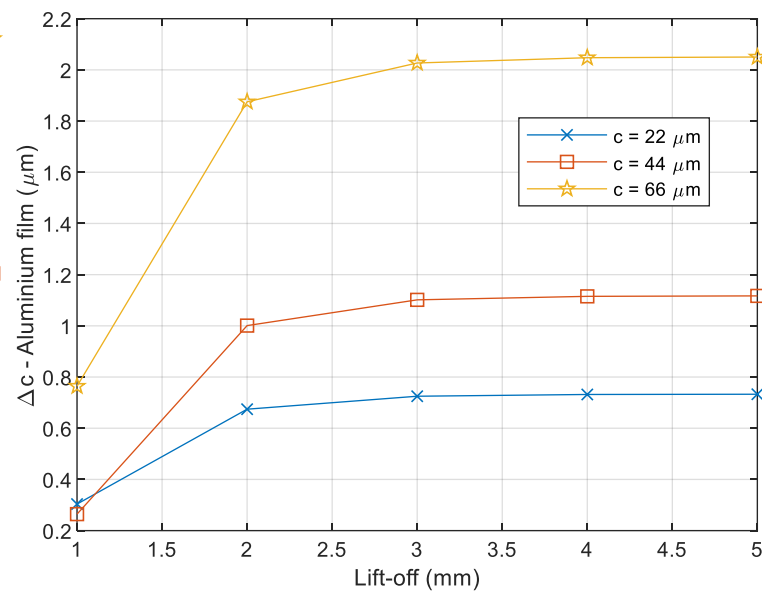

(b)

Fig. 10 Reconstructed thickness of aluminium films under the optimal frequency of $16 \mathrm{kHz}$ with different sensor lift-offs (a) absolute value (b) error with respect to the actual thickness

Since the thickness is found can be accurately reconstructed at the optimal frequency - $16 \mathrm{kHz}$ for both aluminium films with different actual thicknesses and copper films with different radii, it is worth further investigating the lift-off effect on the reconstructed thickness under the optimal working frequency. In Fig. 10, it can be observed that the thickness of aluminium films (for different actual thicknesses) can be reconstructed with a small error of less than $3.5 \%$. Moreover, thickness after the reconstruction is gradually stable with the increased sensor lift-off, with the aluminium film of $66 \mu \mathrm{m}$ the highest absolute reconstruction error. In Fig. 11, a similar trend (reduced and stable error for increased sensor lift-offs) can be seen on the reconstructed thickness of the copper films with different radii. The error of the thickness reconstruction for copper films is around $5 \%$. Moreover, the difference between the thickness curves of different radii is not significant after the reconstruction. In the experimental, the sensor lift-off is selected to be a series of small values of less than $5 \mathrm{~mm}$, since larger sensor lift-off will result in a distorted value due to the extremely weak coupling effect between sensor and sample.

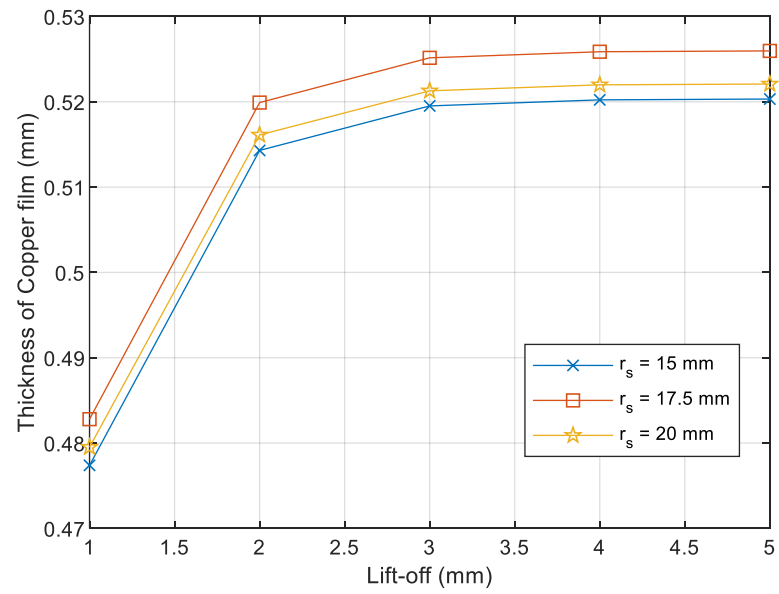

(a)

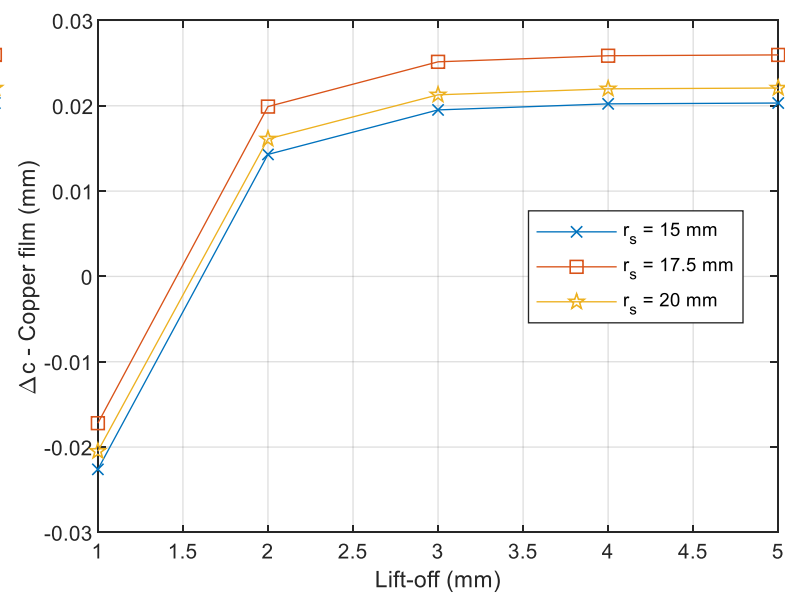

(b)

Fig. 11 Reconstructed thickness of copper films under the optimal frequency of $16 \mathrm{kHz}$ with different sensor lift-offs (a) absolute value (b) error with respect to the actual thickness 


\section{Conclusions}

In this paper, to address the lift-off issue on the thickness measurement of conductive (finite) disk films, a single-frequency algorithm has been proposed based on the eddy current method. By inputting the measured resistance (i.e., the real part of impedance) from two transmitter-receiver sensing pairs, the thickness is retrieved under an optimal working frequency. Unlike the previous peak-frequency feature on the swept-frequency impedance, the proposed method is time-saving and does not need to recalibrate for different sensor setups applied to online real-time measurements. Moreover, instead of directly taking the phase term out of the integration in the Dodd-Deed formulation (only valid on a limited range of thicknesses), a frequency-dependent constant has been found between the imaginary part of the phase $\operatorname{Im}(\phi(\alpha))$ and a series for the real part of phase term $\alpha \operatorname{Re}(\phi(\alpha))$. Therefore, the proposed method is more general for a wider range of thicknesses. Besides, the proposed method has, for the first time, addressed the problem of reducing the lift-off effect on measuring the thickness of a finite-size circular plate using single-frequency data. From the experiment results, it shows that the error of retrieved thickness is less than $5 \%$ for a range of actual thicknesses, radius, and sensor lift-offs.

\section{Acknowledgement}

This work was supported by [UK Engineering and Physical Sciences Research Council (EPSRC)] [grant number: EP/P027237/1] [title: Real-time In-line Microstructural Engineering (RIME)].

\section{References}

1. J. C. Moulder, E. Uzal, and J. H. Rose, "Thickness and conductivity of metallic layers from eddy current measurements," Review of Scientific Instruments, vol. 63, no. 6, 1992.

2. A. Tamburrino, and R. Guglielmo. "Fast methods for quantitative eddy-current tomography of conductive materials." IEEE transactions on magnetics, vol. 42, pp. 2017-2028, 2006.

3. G. Yang, G. Dib, L. Udpa, A. Tamburrino and S. S. Udpa, "Rotating Field EC-GMR Sensor for Crack Detection at Fastener Site in Layered Structures," IEEE Sensors Journal, vol. 15, no. 1, pp. 463-470, Jan. 2015.

4. D. Vasic, V. Bilas and D. Ambrus, "Pulsed eddy-current nondestructive testing of ferromagnetic tubes," IEEE Transactions on Instrumentation and Measurement, vol. 53, no. 4, pp. 1289-1294, Aug. 2004.

5. I. Z. Abidin, C. Mandache, G. Y. Tian and M. Morozov, "Pulsed eddy current testing with variable duty cycle on rivet joints," NDT \& E International, vol. 42, no. 7, pp. 599-605, 2009.

6. M. Lu, et al. "Acceleration of frequency sweeping in eddy-current computation," IEEE Transactions on Magnetics, vol. 53, pp. $1-8,2017$.

7. Y. Shin, D. Choi, Y. Kim and S. Lee, "Signal characteristics of differential-pulsed eddy current sensors in the evaluation of plate thickness," NDT \& E International, vol. 42, no. 3, pp. 215-221, 2009.

8. G. Y. Tian and A. Sophian, "Reduction of lift-off effects for pulsed eddy current NDT," NDT \& E International, vol. 38, no. 4, pp. 319-324, 2005.

9. A. V. Egorov, V. V. Polyakov, D. S. Salita, E. A. Kolubaev, S. G. Psakhie, A. G. Chernyavskii, I. V. Vorobei, "Inspection of aluminum alloys by a multi-frequency eddy current method," Defence Technology, vol. 11, no. 2, pp. 99-103, 2015.

10. W. Yin and A.J. Peyton, "Thickness measurement of non-magnetic plates using multi-frequency eddy current sensors," NDT \& E International, vol. 40, no. 1, pp. 43-48, 2007.

11. W. Zhou, M. Lu et al. "Three-dimensional electromagnetic mixing models for dual-phase steel microstructures," Applied Sciences, vol. 8, pp. 529, 2018.

12. T. Theodoulidis and E. E. Kriezis, "Eddy current canonical problems (with applications to nondestructive evaluation)", Material Science, 2006.

13. G. Tytko and L. Dziczkowski, "E-Cored Coil With a Circular Air Gap Inside the Core Column Used in Eddy Current Testing," IEEE Transactions on Magnetics, vol. 51, no. 9, pp. 1-4, Sept. 2015.

14. W. Yin, S. J. Dickinson, and A. Peyton, "Imaging the continuous conductivity profile within layered metal structures using inductance spectroscopy,” IEEE Sensors Journal, vol. 5, no. 2, pp. 161-166, 2005.

15. M. Lu, H. Xu, W. Zhu, L. Yin et al. "Conductivity Lift-off Invariance and measurement of permeability for ferrite metallic plates,” NDT \& E International, vol. 95, pp. 36-44, Apr. 2018.

16. M. Lu, R. Huang, W. Yin, Q. Zhao, and A. Peyton, "Measurement of permeability for ferrous metallic plates using a novel liftoff compensation technique on phase signature," IEEE Sensors Journal, vol. 19, no. 17, pp. 7440-7446, 1 Sept.1, 2019.

17. M. Lu, et al. "Determination of the magnetic permeability, electrical conductivity, and thickness of ferrite metallic plates using a multi-frequency electromagnetic sensing system," IEEE Transactions on Industrial Informatics, vol. 15, pp. 4111-4119, 2019.

18. I. N. Prassianakis and N. I. Prassianakis, "Ultrasonic testing of non-metallic materials: concrete and marble," Theoretical and Applied Fracture Mechanics, vol. 42, no. 2, pp. 191-198, Nov. 2004.

19. J. Kral, R. Smid, H. M. G. Ramos, and A. L. Ribeiro, "The lift-off effect in eddy currents on thickness modeling and measurement," IEEE Transactions on Instrumentation and Measurement, vol. 62, pp. 2043-2049, 2013.

20. C. C. Tai, J. H. Rose, and J. C. Moulder, "Thickness and conductivity of metallic layers from pulsed eddy-current measurements," Review of Scientific Instruments, vol. 67, no. 11, 3965-3972, 1996.

21. H. Wang, W. Li, and Z. Feng, "Noncontact thickness measurement of metal films using eddy-current sensors immune to distance variation," IEEE Transactions on Instrumentation and Measurement, vol. 64, no. 9, pp. 2557-2564, 2015.

22. E. Pinotti and E. Puppin, "Simple Lock-In Technique for Thickness Measurement of Metallic Plates," IEEE Transactions on Instrumentation and Measurement, vol. 63, no. 2, pp. 479-484, 2014. 
23. C. Mandache, and J. H. V. Lefebvre, "Transient and harmonic eddy currents: Lift-off point of intersection." NDT \& E International, vol. 39, no. 1, pp. 57-60, 2006.

24. M. Fan, et al. "Thickness measurement using liftoff point of intersection in pulsed eddy current responses for elimination of liftoff effect," Sensors and Actuators A: Physical, vol. 251, pp. 66-74, 2016.

25. C. S. Angani et al. "Lift-off point of intersection feature in transient eddy-current oscillations method to detect thickness variation in stainless steel." IEEE Transactions on Magnetics, vol. 52, no.6, pp.1-8, 2016.

26. J.R.S. Avila, M. Lu et al. "Accurate measurements of plate thickness with variable lift-off using a combined inductive and capacitive sensor,” NDT \& E International, vol. 110, pp. 102202, 2020.

27. M. Lu, L. Yin, A. J. Peyton and W. Yin, "A novel compensation algorithm for thickness measurement immune to lift-off variations using eddy current method," IEEE Transactions on Instrumentation and Measurement, vol. 65, no. 12, pp. 2773-2779, Dec. 2016.

28. M. Lu, X. Meng, W. Yin, Z. Qu, F. Wu, J. Tang, et al., "Thickness measurement of non-magnetic steel plates using a novel planar triple-coil sensor,” NDT \& E International, vol. 107, 2019.

29. R. Huang, M. Lu, A. Peyton, and W. Yin, "Thickness measurement of metallic plates with finite planar dimension using eddy current method," IEEE Transactions on Instrumentation and Measurement, early access, 2020. Doi:10.1109/TIM.2020.2987413.

30. M. Lu et al. "Measurement of ferromagnetic slabs permeability based on a novel planar triple-coil sensor," IEEE Sensors J., vol. 20, no. 6, pp. 2904-2910, 2020.

31. M. Lu, W. Zhu, L. Yin, A. J. Peyton, W. Yin, and Z. Qu, "Reducing the lift-off effect on permeability measurement for magnetic plates from multifrequency induction data," IEEE Transactions on Instrumentation and Measurement, vol. 67, no. 1, pp. 167-174, Jan. 2018

32. C. V. Dodd, and W. E. Deeds, “Analytical solutions to eddy-current probe-coil problems,” Journal of applied physics, vol. 39, no. 6, pp. 2829-2838, 1968 .

33. R. Huang, M. Lu et al, "Measurement of the radius of metallic plates based on a novel finite region eigenfunction expansion (FREE) method,” IEEE Sensors Journal, vol. 20, pp. 15099 - 15106, 2020. Doi: 10.1109/JSEN.2020.3009443.

34. R. Huang, M. Lu et al, "Measuring co-axial hole size of finite-size metallic disk based on a dual-constraint integration feature using multi-frequency eddy current testing," IEEE Transactions on Instrumentation and Measurement, vol. 70, pp. 1-7, 2020. Doi: 10.1109/TIM.2020.3026762.

35. M. Lu et al, "Thickness Measurement of Metallic Film Based on a High-Frequency Feature of Triple-Coil Electromagnetic Eddy Current Sensor," IEEE Transactions on Instrumentation and Measurement, vol. 70, pp. 1-8, 2020. Doi: 10.1109/TIM.2020.3027929.

36. M. Lu et al, "Measuring Lift-Off Distance and Electromagnetic Property of Metal Using Dual-Frequency Linearity Feature," IEEE Transactions on Instrumentation and Measurement, vol. 70, pp. 1-9, 2020. Doi: 10.1109/TIM.2020.3029348.

37. M. Lu et al, "Lift-off tolerant pancake eddy-current sensor for the thickness and spacing measurement of non-magnetic plates," IEEE Transactions on Instrumentation and Measurement, early access, 2020. Doi: 10.1109/TIM.2020.3033377.

38. Chew, W. C. Waves and Fields in Inhomogenous Media. New York, NY, USA: IEEE Press, 1995, chapter. 2, pp. 4-10.

39. M. Lu et al, "Inversion of distance and magnetic permeability based on material-independent and lift-off insensitive algorithms using eddy current sensor," IEEE Transactions on Instrumentation and Measurement, early access, 2020. Doi: 10.1109/TIM.2020.3036099.

40. W. Yin et al, "Measurements of Thickness for Metallic Plates With Co-Axial Holes Using a Novel Analytical Method With the Modified Integration Range," IEEE Access, vol. 8, pp. 198301 - 198306, 2020.

41. X. Meng, M. Lu et al, "Inversion of lift-off distance and thickness for non-magnetic metal using eddy current testing," IEEE Transactions on Instrumentation and Measurement, vol. 70, 2020. Doi: 10.1109/TIM.2020.3038289.

42. M. Lu, X. Meng, et al, "Determination of surface crack orientation based on thin-skin regime using triple-coil drive-pickup eddycurrent sensor," IEEE Transactions on Instrumentation and Measurement, early access, 2020. Doi: 10.1109/TIM.2020.3044729.

43. Yin, W.; Tang, J.; Lu, M.; et al. An equivalent-effect phenomenon in eddy current non-destructive testing of thin structures. IEEE Access, 2019, 7, pp. 70296-70307.

44. Lu, M.; et al. Determination of Surface Crack Orientation Based on Thin-Skin Regime Using Triple-Coil Drive-Pickup EddyCurrent Sensor. IEEE Transactions on Instrumentation and Measurement, 2020, 70, pp. 1-9. DOI: 10.1109/TIM.2020.3044729

45. M. Lu, et al., "Prediction of the asymptotical magnetic polarization tensors for cylindrical samples using the boundary element method," In 2015 IEEE Sensors Applications Symposium (SAS), pp. 1-4. IEEE, 2015.

46. R. Huang, M. Lu, A. Peyton, and W. Yin, "A novel perturbed matrix inversion based method for the acceleration of finite element analysis in crack-scanning eddy current NDT," IEEE Access, vol. 8, pp. 12438-12444, 2020.

47. J. Tang et al., "A Novel Efficient FEM Thin Shell Model for Bio-Impedance Analysis," Biosensors, vol. 10, no. 6, pp. 69, 2020.

48. L. Chen, et al., "Textile Based Capacitive Sensor for Physical Rehabilitation via Surface Topological Modification," ACS Nano, vol. 14, no. 7, pp. 8191-8201, 2020. DOI: 10.1021/acsnano.0c01643

49. Z. Jin, et al., "Methods of Controlling Lift-off in Conductivity Invariance Phenomenon for Eddy Current Testing," IEEE ACCESS, vol. 8, pp. 2169-3536, 2020. DOI: 10.1109/ACCESS.2020.3007216.

50. J. Tang, et al., "Effect of frozen-thaw injury on cell membrane and bio-impedance," In 2020 IEEE International Instrumentation and Measurement Technology Conference (I2MTC), pp. 1-6. IEEE, 2020.

51. J. Tang, et al., "Bio-impedance spectroscopy for frozen-thaw of bio-samples: Non-contact inductive measurement and finite element (FE) based cell modelling," Journal of Food Engineering, vol. 272, pp. 109784, 2020.

52. H. Xu et al., "Imaging a weld cross-section using a novel frequency feature in multi-frequency eddy current testing," InsightNon-Destructive Testing and Condition Monitoring, vol. 61, no. 12, pp. 738 - 743, 2019.

53. Y. Xie et al., "Novel Wearable Sensors for Biomechanical Movement Monitoring Based on Electromagnetic Sensing Techniques," IEEE Sensors Journal, vol. 20, no. 2, 2020. DOI: 10.1109/JSEN.2019.2943487

54. W. Yin et al., "Permeability invariance phenomenon and measurement of electrical conductivity for ferrite metallic plates," Insight-Non-Destructive Testing and Condition Monitoring, vol. 61, no. 8, pp. 472 - 479, 2019.

55. M. Lu et al., "A model for the triboelectric nanogenerator with inductive load and its energy boost potential," Nano Energy, vol. 63, pp. 103883, 2019.

56. M. Lu et al., "Forward solver for deep earth exploration and induction logging using custom built Edge-Element FEM technique," Acta Geologica Sinica, vol. 93, pp. 302-304, 2019.

57. L. Chen et al., "Whole System Design of Wearable Magnetic Induction Sensor for Physical Rehabilitation," Advanced Intelligent Systems, vol. 1, no. 1, pp. 1900037, 2019. 
58. Y. X et al., "A self-powered radio frequency (RF) transmission system based on the combination of triboelectric nanogenerator (TENG) and piezoelectric element for disaster rescue/relief," Nano Energy, vol. 54, pp. 331-340, 2018.

59. W. Yin et al., "Custom edge-element FEM solver and its application to eddy-current simulation of realistic 2M-element human brain phantom," Bioelectromagnetics, vol. 39, no. 8, pp. 604-616, 2018.

60. L. Yin et al., "Detection of corrosion pits based on an analytically optimised eddy current sensor," Insight-Non-Destructive Testing and Condition Monitoring, vol. 60, no. 10, pp. 561-567, 2018.

61. W. Yin et al., "Acceleration of eddy current computation for scanning probes," Insight-Non-Destructive Testing and Condition Monitoring, vol. 60, no. 10, pp. 547-555, 2018.

62. W. Zhou et al., "Three-dimensional electromagnetic mixing models for dual-phase steel microstructures," Applied Sciences, vol. 8, no. 4, pp. 547-555, 2018.

63. M. Lu, et al., "Determining the magnetic permeability of ferrite steel strip by a custom inversion method," In Proc. 12th ECNDT, pp. 1-8. 2018.

64. J. Tang, et al., "Cellular structure analysis based on magnetic induction finite element method simulations and measurements," bioRxiv, pp. 275271, 2018. DOI: 10.1101/275271

65. J.R.S. Avila, et al., "A novel dual modality sensor with sensitivities to permittivity, conductivity, and permeability," IEEE Sensors Journal, vol. 18, no. 1, pp. 356-362, 2017.

66. T. Yang, et al., "Level measurement for saline with a small surface area using high frequency electromagnetic sensing technique," Measurement, vol. 101, pp. 118-125, 2017.

67. X. Meng, et al, "Evaluation of coating thickness using lift-off insensitivity of eddy current sensor," Sensors, vol. 21, no. 2, pp. 419, 2021.

68. Hu, G.; Huang, R.; Lu, M.; Zhou, L.; Yin, W. Measurement of Radius of a Metallic Ball Using Eddy Current Testing Based on Peak Frequency Difference Feature. Preprints 2021, 2021070220 (doi: 10.20944/preprints202107.0220.v2).

69. M. Lu, et al., "Lift-off invariant inductance of steels in multi-frequency eddy-current testing." NDT \& E International, vol. 121, p. $102458,2021$.

70. Z. Jin, et al., "Boundary-element analysis of magnetic polarization tensor for metallic cylinder." IEEE Access, vol. 9, pp. 6325063256, 2021. 